\title{
A Novel Way of Treating Multidrug-resistant Enterococci
}

\author{
Hem Desai, Ryan Wong, Ahmed Khurshid Pasha \\ Department of Internal Medicine, University of Arizona College of Medicine, Tucson, Arizona, USA
}

\begin{abstract}
Context: Daptomycin is the only antibiotic available with in vitro bactericidal activity against vancomycin-resistant enterococci (VRE). Its increased use has resulted in cases of decreased daptomycin efficacy. Recent in vitro studies have shown effective use of beta $(\beta)$-lactam and daptomycin antibiotics, as a combination therapy, in the treatment of VRE. We describe a case of effective treatment in a patient with VRE infection using dual ampicillin and daptomycin therapy that shows bench-to-bedside application of the abovementioned finding. Case Report: A 76-year-old gentleman with a history of bilateral arthroplasty was admitted with a swollen left knee. Blood cultures were positive for Enterococcus faecium. Left knee joint aspiration showed leukocytosis and alpha defensins. Extensive imaging did not show any other source of infection. Culture sensitivity results showed multidrug-resistant enterococci sensitive to daptomycin. The patient was started on intravenous (IV) daptomycin. His left knee prosthesis was explanted and a spacer was placed. The patient continued to be bacteremic for 10 days after removing the knee prosthesis. The patient was trialed on combination IV ampicillin and daptomycin. His blood culture turned negative 2 days later. The patient was discharged home to continue 6 weeks of IV ampicillin and daptomycin. Conclusion: The exact mechanism of the daptomycin/ampicillin synergy effect is unclear. Current hypothesis suggests that ampicillin causes a reduction in the net positive charge of the bacterial surface, possibly by releasing lipoteichoic acid (LTA) from the cell wall. This process increases the ability of the cationic daptomycin/calcium complex to bind to the cell wall more effectively. Our case shows the clinical application of the same. A prospective randomized control trial to explore the effectiveness of dual antibiotic therapy in vivo is needed. If proven, daptomycin/ $\beta$-lactam can become a standard of care to treat VRE and decrease daptomycin nonsusceptibility.
\end{abstract}

Keywords: Daptomycin/beta ( $\beta$ )-lactam, daptomycin nonsusceptibility, vancomycin-resistant enterococci

Address for correspondence: Dr. Hem Desai, 3300 N Paseo De Los Rios, Apartment 22204, Tucson - 85712, Arizona, USA.

E-mail: hdesai@deptofmed.arizona.edu

\section{Context}

Accounting for almost $10 \%$ of total bloodstream infections, Enterococcus is the fourth most common pathogen responsible for bloodstream infections in North America. ${ }^{[1]}$ Of those total number of Enterococcus infections, $25 \%$ are resistant to vancomycin. ${ }^{[2]}$ Vancomycin-resistant enterococci (VRE) infections have been associated with increased mortality and morbidity. ${ }^{[3,4]}$ In patients infected with VRE, the

\begin{tabular}{|l|l|}
\hline \multicolumn{2}{|c|}{ Access this article online } \\
\hline Quick Response Code: & Website: \\
\hline & www.najms.org \\
\cline { 2 - 2 } & \\
\hline
\end{tabular}

odds of death are 2.5 times higher compared with vancomycin-susceptible enterococci. ${ }^{[3]}$ Most of these antibiotics are bacteriostatic and associated with extensive drug-drug interactions. ${ }^{[5-7]}$ Daptomycin is the only antibiotic available with in vitro bactericidal activity against VRE. ${ }^{[8-10]}$ Daptomycin is being increasingly used, especially in the critically ill and immunosuppressed, even though it is not approved by the Food and Drug Administration (FDA). ${ }^{[11]}$ There have been reports of

\footnotetext{
This is an open access article distributed under the terms of the Creative Commons Attribution-NonCommercial-ShareAlike 3.0 License, which allows others to remix, tweak, and build upon the work non-commercially, as long as the author is credited and the new creations are licensed under the identical terms.
}

For reprints contact: reprints@medknow.com

How to cite this article: Desai $\mathrm{H}$, Wong R, Ahmed K. A novel way of treating multidrug-resistant enterococci. North Am J Med Sci 2016;8:229-31. 
daptomycin nonsusceptible enterococci emergence during monotherapy. ${ }^{[12,13]}$ Studies have shown that $2-3 \%$ of VRE cases may be nonsusceptible to daptomycin. ${ }^{[13]}$ In those cases of resistant organisms, there are no other antibiotic treatment options. There have been recent in vitro studies showing effective use of beta $(\beta)$-lactams and daptomycin on daptomycin nonsusceptible enterococci. ${ }^{[14-16]}$ We describe a case of effective treatment in a patient with vancomycin-resistant Enterococcus faecium with dual ampicillin and daptomycin therapy that shows bench-to-bedside application of the abovementioned finding.

\section{Case Presentation}

A 76-year-old gentleman with a past medical history significant for extensive coronary artery disease and bilateral arthroplasty was admitted with the complaint of left knee swelling. He was afebrile on presentation. His left knee was erythematous, tender, and swollen compared to the right knee. Arthrocentesis was positive for alpha defensins with white blood cell (WBC) count of 18,000/uL. Fluid culture was negative. No crystalopathy was identified. Blood cultures were positive for Enterococci faecium. Transesophageal echocardiogram (TEE) was performed to rule out infective endocarditis and was negative.

The VRE, found in blood culture, were sensitive to daptomycin with 1:2 dilutions and resistant to ampicillin with 1:32 dilution. The patient was started on intravenous (IV) daptomycin $6 \mathrm{mg} / \mathrm{kg}$ daily. His left knee prosthesis was explanted and a spacer was placed on the fourth day of admission. Blood cultures were performed every other day to assess the antibiotic response. His blood cultures continued to grow VRE for 10 days after the explantation of the prosthesis. Extensive imaging was performed to find the source of infection. A whole body nuclear-tagged WBC scan did not show any signs of persistent infection. Magnetic resonance imaging (MRI) of the chest, abdomen, and pelvis with and without contrast failed to show any focus of infection. Arthrocentesis of the right knee and repeat TEE were negative as well. Due to persistent bacteremia, the patient was tried on a combination of IV ampicillin and daptomycin. On the second day of the combined therapy, the next set of blood cultures were obtained and were negative for the first time since admission. Repeat blood cultures continued to be negative for 7 more days. He was eventually discharged with the peripherally inserted central catheter (PICC) line to continue 6 weeks of IV ampicillin and daptomycin.

\section{Discussion}

This case showed an interesting clinical application of combination ampicillin and daptomycin to combat daptomycin nonsusceptible organisms. This phenomenon had been described mostly in in vitro studies.

Sakoulas et al. demonstrated a threefold decrease in daptomycin minimum inhibitory concentration (MIC), when VRE strain was grown in a medium containing $50 \mathrm{mg} / \mathrm{L}$ ampicillin and $100 \mathrm{mg} / \mathrm{L}$ ampicillin. ${ }^{[14]}$ However, the exact mechanism of the daptomycin/ampicillin synergistic effect is unclear at this time. The current hypothesis suggested that ampicillin causes a reduction in the net positive charge of the bacterial surface by releasing lipoteichoic acid (LTA) from the cell wall. The loss of LTA released cell autolysins that caused bacterial cell wall destabilization. More importantly, the reduction in cell wall positive charge appeared to allow the cationic daptomycin/calcium complex to bind more effectively to the cell wall. In a fluorescence-labeled daptomycin assay, this mechanism was supported as ampicillin pretreatment that resulted in an increase in daptomycin binding. ${ }^{[14,16]}$

Although, difficult to prove that in the current case, the clearance of bacteremia was related to the abovementioned mechanism, the patient continued to be bacteremic for 10 days while being treated with daptomycin monotherapy. With the addition of ampicillin, blood cultures drawn a couple of days after were negative and remained negative. This was similarly described in the report by Sakoulas et al., where a patient with persistent bacteremia refractory to 7 days of daptomycin/linezolid cleared the infection within $24 \mathrm{~h}$ of starting dual high-dose daptomycin and ampicillin. ${ }^{[14]}$ In a multiple hospitals study, Moise et al . showed a significantly higher daptomycin monotherapy failure when daptomycin MIC values were 3-4 $\mu \mathrm{g} / \mathrm{L}$, compared to MIC $<2 \mu \mathrm{g} / \mathrm{L}(P=0.005)$. When comparing the two different MIC groups in patients treated with $\beta$-lactam/daptomycin dual therapy, treatment failure was not significantly different $(P=0.417) \cdot{ }^{[15]}$ This may be evidence to suggest that adjunct ampicillin may be indicated in infections where daptomycin MIC is 3-4.

Additionally, our case shows that in vitro drug susceptibility testing may not accurately translate into clinical potency. Even though the enterococcus isolate was resistant to ampicillin, the addition of ampicillin resulted in effective clearance of the patient's bacteremia, increasing the in vivo potency of daptomycin.

Other studies have described increased daptomycin efficacy with the addition of ceftaroline, a cephalosporin to which enterococci are intrinsically resistant. ${ }^{[17]}$ Dual antibiotic therapy with ampicillin and daptomycin additionally appeared to be effective in treating methicillin-resistant staphylococcus aureus. ${ }^{[18]}$ 


\section{Conclusion}

Daptomycin is a potent bactericidal antibiotic. With the increased prevalence of VRE related infections, daptomycin may be the last line of defense against enterococci. Increasing prevalence of daptomycin nonsusceptible enterococci presents an added challenge for treating such life-threatening infections. A few in vitro experiments have studied the increased efficacy of daptomycin in the presence of $\beta$-lactam antibiotics. A prospective randomized control trial to explore the effectiveness of dual antibiotic therapy in vivo is needed. Further studies to assess synergistic effect of different antibiotics with daptomycin should be assessed. As multidrug-resistant organisms are becoming more common, it would be worthwhile to investigate synergistic antibiotic therapy. If proven, daptomycin/ $\beta$-lactam can become a standard of care to treat VRE and can decrease daptomycin nonsusceptibility.

\section{Financial support and sponsorship}

Nil.

\section{Conflicts of interest}

There are no conflicts of interest.

\section{References}

1. Deshpande LM, Fritsche TR, Moet GJ, Biedenbach DJ, Jones RN. Antimicrobial resistance and molecular epidemiology of vancomycin-resistant enterococci from North America and Europe: A report from the SENTRY antimicrobial surveillance program. Diagn Microbiol Infect Dis 2007;58:163-70.

2. Murray BE. Vancomycin-resistant enterococcal infections. N Engl J Med 2000;342:710-21.

3. DiazGranados CA, Zimmer SM, Klein M, Jernigan JA. Comparison of mortality associated with vancomycin-resistant and vancomycin-susceptible enterococcal bloodstream infections: A meta-analysis. Clin Infect Dis 2005;41:327-33.

4. Ramsey AM, Zilberberg MD. Secular trends of hospitalization with vancomycin-resistant enterococcus infection in the United States, 2000-2006. Infect Control Hosp Epidemiol 2009;30:184-6.

5. Gerson SL, Kaplan SL, Bruss JB, Le V, Arellano FM, Hafkin B, et al. Hematologic effects of linezolid: Summary of clinical experience. Antimicrob Agents Chemother 2002;46:2723-6.
6. Ramsey TD, Lau TT, Ensom MH. Serotonergic and adrenergic drug interactions associated with linezolid: A critical review and practical management approach. Ann Pharmacother 2013;47:543-60.

7. Olsen KM, Rebuck JA, Rupp ME. Arthralgias and myalgias related to quinupristin-dalfopristin administration. Clin Infect Dis 2001;32:e83-6.

8. Poutsiaka DD, SkiffingtonS, Miller KB, HadleyS, Snydman DR. Daptomycin in the treatment of vancomycin-resistant Enterococcus faecium bacteremia in neutropenic patients. J Infect 2007;54:567-71.

9. Rybak MJ, Hershberger E, Moldovan T, Grucz RG. In vitro activities of daptomycin, vancomycin, linezolid, and quinupristin-dalfopristin against Staphylococci and Enterococci, including vancomycin-intermediate and-resistant strains. Antimicrob Agents Chemother 2000;44:1062-6.

10. Alder JD. Daptomycin: A new drug class for the treatment of Gram-positive infections. Drugs Today (Barc) 2005;41:81-90.

11. Whang DW, Miller LG, Partain NM, McKinnell JA. Systematic review and meta-analysis of linezolid versus daptomycin for treatment of vancomycin-resistant enterococcal bloodstream infections. Antimicrob Agents Chemother 2013;57:5013-8.

12. Lewis JS $2^{\text {nd }}$, Owens A, Cadena J, Sabol K, Patterson JE, Jorgensen JH. Emergence of daptomycin resistance in Enterococcus faecium during daptomycin therapy. Antimicrob Agents Chemother 2005;49:1664-5.

13. Kelesidis T, Humphries R, Uslan DZ, Pegues DA. Daptomycin nonsusceptible enterococci: An emerging challenge for clinicians. Clin Infect Dis 2011;52:228-34.

14. Sakoulas G, Bayer AS, Pogliano J, Tsuji BT, Yang SJ, Mishra NN, et al. Ampicillin enhances daptomycin- and cationic host defense peptide-mediated killing of ampicillin- and vancomycin-resistant Enterococcus faecium. Antimicrob Agents Chemother 2012;56:838-44.

15. Moise PA, Sakoulas G, McKinnell JA, Lamp KC, DePestel DD, Yoon MJ, et al. Clinical outcomes of daptomycin for vancomycin-resistant enterococcus bacteremia. Clin Ther 2015;37:1443-53.e2.

16. Bravetti AL, Mesnage S, Lefort A, Chau F, Eckert C, Garry L, et al. Contribution of the autolysin AtlA to the bactericidal activity of amoxicillin against Enterococcus faecalis JH2-2. Antimicrob Agents Chemother 2009;53:1667-9.

17. Rose WE, Schulz LT, Andes D, Striker R, Berti AD, Hutson PR, et al. Addition of ceftaroline to daptomycin after emergence of daptomycin-nonsusceptible Staphylococcus aureus during therapy improves antibacterial activity. Antimicrob Agents Chemother 2012;56:5296-302.

18. Rand KH, Houck HJ. Synergy of daptomycin with oxacillin and other beta-lactams against methicillin-resistant Staphylococcus aureus. Antimicrob Agents Chemother 2004;48:2871-5 